\title{
Quantum Delayed-Choice Experiment with a Beam Splitter in a Quantum Superposition
}

\author{
Shi-Biao Zheng, ${ }^{1, *}$ You-Peng Zhong, ${ }^{2}$ Kai Xu, ${ }^{2}$ Qi-Jue Wang, ${ }^{2}$ H. Wang, ${ }^{2}$ Li-Tuo Shen, ${ }^{1}$ Chui-Ping Yang, ${ }^{3}$ \\ John M. Martinis, ${ }^{4, \dagger}$ A. N. Cleland, ${ }^{5, \$}$ and Si-Yuan Han ${ }^{6,7}$ \\ ${ }^{1}$ Department of Physics, Fuzhou University, Fuzhou 350116, China \\ ${ }^{2}$ Department of Physics, Zhejiang University, Hangzhou 310027, China \\ ${ }^{3}$ Department of Physics, Hangzhou Normal University, Hangzhou 310036, China \\ ${ }^{4}$ Department of Physics, University of California, Santa Barbara, California 93106, USA \\ ${ }^{5}$ Institute for Molecular Engineering, University of Chicago, Chicago, Illinois 60637, USA \\ ${ }^{6}$ Department of Physics and Astronomy, University of Kansas, Lawrence, Kansas 66045, USA \\ ${ }^{7}$ Beijing National Laboratory for Condensed Matter Physics, Institute of Physics, Chinese Academy of Sciences, Beijing 100190, China
}

(Received 11 August 2015; published 28 December 2015)

\begin{abstract}
A quantum system can behave as a wave or as a particle, depending on the experimental arrangement. When, for example, measuring a photon using a Mach-Zehnder interferometer, the photon acts as a wave if the second beam splitter is inserted, but as a particle if this beam splitter is omitted. The decision of whether or not to insert this beam splitter can be made after the photon has entered the interferometer, as in Wheeler's famous delayed-choice thought experiment. In recent quantum versions of this experiment, this decision is controlled by a quantum ancilla, while the beam splitter is itself still a classical object. Here, we propose and realize a variant of the quantum delayed-choice experiment. We configure a superconducting quantum circuit as a Ramsey interferometer, where the element that acts as the first beam splitter can be put in a quantum superposition of its active and inactive states, as verified by the negative values of its Wigner function. We show that this enables the wave and particle aspects of the system to be observed with a single setup, without involving an ancilla that is not itself a part of the interferometer. We also study the transition of this quantum beam splitter from a quantum to a classical object due to decoherence, as observed by monitoring the interferometer output.
\end{abstract}

DOI: 10.1103/PhysRevLett.115.260403

PACS numbers: 03.65.Ta, 42.50.Dv, 42.50.St

The wave-particle duality is one of the fundamental mysteries that lie at the heart of quantum mechanics. However, these two incompatible aspects cannot be observed simultaneously, as captured by Bohr's principle of complementarity [1-5]: Particlelike versus wavelike outcomes are selected by experimental arrangements that are mutually exclusive. This is well illustrated by the Mach-Zehnder interferometer, as shown in Fig. 1(a). Split by the first beam splitter $\left(\mathrm{BS}_{1}\right)$, a photon travels along two paths, 0 and 1. The relative phase $\theta$ between the quantum states associated with these paths is tunable. In the presence of the second beam splitter $\left(\mathrm{BS}_{2}\right)$, the two paths are recombined and the probability for detecting the photon in the detector $D_{0}$ or $D_{1}$ is a sinusoidal function of $\theta$ exhibiting wavelike interference fringes. On the other hand, in the absence of $\mathrm{BS}_{2}$, the experiment reveals which path the photon followed, and the photon is detected in one or the other detector with equal probability $1 / 2$, thus behaving as a particle.

One can argue that the behavior of the photon is predetermined by the experimental arrangement, where the presence or absence of the second beam splitter affects the photon prior to its entering the interferometer. The possibility of such a causal link is precluded in Wheeler's delayed-choice experiment [6-8], in which the observer randomly chooses whether to insert $\mathrm{BS}_{2}$, and thus whether to perform an interference or a which-path experiment, after the photon has passed through $\mathrm{BS}_{1}$. Therefore, the photon could not "know" in advance which behavior it should exhibit. Wheeler's delayed-choice experiment has been demonstrated previously [9-13], where the spacelike separation between the setup selection and the photon entry into the interferometer was achieved in Ref. [12]. Recently, a quantum version of the delayed-choice experiment was suggested [14] in which the action of $\mathrm{BS}_{2}$ is controlled by a quantum ancilla. The scheme is illustrated in Fig. 1(b), where each beam splitter is replaced by a Hadamard operation $H$, and the second Hadamard operation is conditionally applied following the phase shift $\theta$. The quantum system exhibits wavelike behavior if the ancilla is in its $|1\rangle$ state and the second Hadamard is applied; if the ancilla is instead in $|0\rangle$, the second Hadamard is not performed, and the system displays particlelike behavior. When the ancilla is prepared in a superposition of $|0\rangle$ and $|1\rangle$, the result is a superposition of wavelike and particlelike states, entangled with the ancilla [14]. This clearly precludes the system knowing in advance which setup will be selected; the quantum superposition effectively replaces the need for Wheeler's spacelike separation of the photon entry into the interferometer and the measurement selection. Instead, the wavelike and particlelike behaviors are postselected by measuring the ancilla in its $(|0\rangle,|1\rangle)$ basis. This allows the complementary wave and particle behaviors to emerge 
(a)

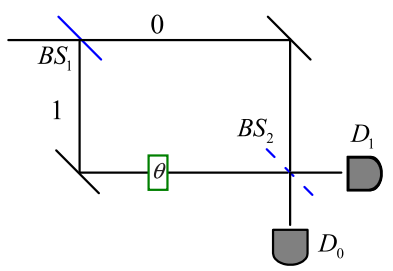

(b)

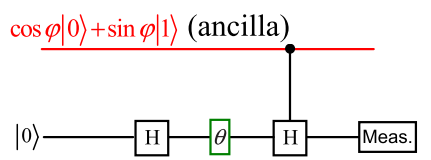

(c)

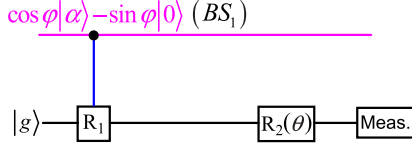

FIG. 1 (color online). (a) Schematic of Wheeler's delayedchoice thought experiment. A photon's state is transformed by the first beam splitter, $\mathrm{BS}_{1}$, into a superposition of two paths, 0 and 1, followed by a tunable phase shift $\theta$. While the photon is inside the interferometer, the observer decides whether or not to insert a second beam splitter, $\mathrm{BS}_{2}$, thereby delaying the decision of whether to measure an interference pattern (wave aspect) or to obtain which-path information (particle aspect), as revealed by the probability of detecting the photon at detector $D_{0}$ and $D_{1}$ as a function of $\theta$. (b) Schematic of the quantum delayed-choice experiment using a controlling quantum ancilla. The effect of each beam splitter is represented by a Hadamard operation, $H$, with the relative phase shift $\theta$ occurring in between these two operations. The second Hadamard is controlled by the ancilla, which can be prepared in a superposition state, allowing simultaneous preparation of the wave and particle aspects of the test system. (c) Schematic of the delayed-choice experiment, implemented with a superconducting beam splitter that is put into a catlike superposition state. The test qubit, initially in the ground state $|g\rangle$, is subjected to two operations, $R_{1}$ and $R_{2}(\theta)$, equivalent in combination to the two Hadamard operations plus the phase shift $\theta . R_{1}$ is implemented ( $\mathrm{BS}_{1}$ active) when the test qubit interacts with a superconducting resonator occupied by a classical coherent field $|\alpha\rangle . R_{2}(\theta)$ is implemented by a classical microwave pulse. The final probability of measuring the qubit in the ground state $|g\rangle$ or excited state $|e\rangle$, for active $\mathrm{BS}_{1}$, then depends on the relative phase $\theta$, thus exhibiting Ramsey interference. When the superconducting resonator is instead in the ground state $|0\rangle$, $R_{1}$ is not implemented ( $\mathrm{BS}_{1}$ inactive), and no $\theta$-dependent Ramsey interference occurs. The wave and particle aspects of the qubit can be superposed by preparing the resonator in a quantum superposition of filled $|\alpha\rangle$ and empty $|0\rangle$ states, generating an output state that encodes both wavelike and particlelike behavior.

from a single experimental setup, showing that the complementarity really resides in the experimental data, rather than resulting from the experiment's physical arrangement.

Quantum delayed-choice experiments have been performed in both NMR [15,16] and optical [17-19] systems. In the optical experiments reported in Refs. $[18,19]$, the quantum correlation between the test photon and the quantum ancilla was verified by the violation of a Bell

inequality; we note that these measurements are subject to the fair-sampling assumption: that is, the large number of coincident photon pairs that fail to be registered are assumed to obey the same statistics as those that are recorded.

Here, we theoretically propose and experimentally implement a variant of the quantum delayed-choice experiment, using a superconducting Ramsey interferometer and the process shown in Fig. 1(c); we note that the Ramsey and Mach-Zehnder interferometers are physically different, but they actually implement the same quantum circuit [20]. In our interferometer, a two-level quantum system (a superconducting qubit) with ground state $|g\rangle$ and excited state $|e\rangle$, which correspond to the paths 0 and 1 in the optical interferometer, is subjected to two Ramsey rotations, $R_{1}$ and $R_{2}(\theta)$, which correspond to the two beam splitters of the Mach-Zehnder interferometer but are induced by microwave fields. The tunable phase shift $\theta$ between the two interfering paths is incorporated into the microwave field that produces $R_{2}(\theta) . R_{1}$ is generated by the microwave field stored in a superconducting resonator. If the resonator is "filled" by exciting it into the appropriate microwave coherent state $|\alpha\rangle$, the qubit will undergo a Hadamard transformation by interacting with this field. This rotates the input state from $|g\rangle$ into a superposition of $|g\rangle$ and $|e\rangle$, producing two paths in the Hilbert space, as with the first beam splitter in the Mach-Zehnder interferometer. These two paths are then recombined by $R_{2}(\theta)$, and that recombination results in $\theta$-dependent wavelike interference fringes in the probabilities of $|g\rangle$ and $|e\rangle$. If the resonator is instead "empty," i.e., in its ground state $|0\rangle$, no rotation $R_{1}$ occurs, and the qubit remains in $|g\rangle$. The second rotation $R_{2}(\theta)$ then produces a superposition of $|g\rangle$ and $|e\rangle$, but the probabilities of $|g\rangle$ and $|e\rangle$ have no $\theta$ dependence, corresponding to particlelike behavior. We note that the required conditional dynamics cannot be realized when the order of the two rotations in Fig. 1(c) is inverted: To realize the resonatorstate-dependent rotation, the qubit should be in the state $|g\rangle$ before interaction with the resonator, so that the resonator's ground state $|0\rangle$ cannot induce any rotation on the qubit; however, when the unconditional rotation $R_{2}(\theta)$ is applied first, before the qubit-resonator interaction the qubit has a probability of $1 / 2$ to be excited by $R_{2}(\theta)$ to the state $|e\rangle$, to which the resonator's ground state can produce a rotation through the vacuum Rabi oscillation [21].

The wave and particle behavior of the qubit can be investigated simultaneously by preparing the resonator in a superposition of its filled $|\alpha\rangle$ and empty $|0\rangle$ states, i.e., in a cat superposition state [22]. The nonclassical nature of this quantum beam splitter (QBS) can be revealed by measuring the negative values of its Wigner function [23], which is the resonator's quasiprobability distribution in phase space. This enables one to verify the existence of a coherent quantum superposition of the filled and empty, or active and inactive, states of the QBS, without performing a Bell 
measurement. In this case, after $R_{2}$, the wave and particle states of the qubit are entangled with the state of the resonator. This is in contrast to previous realizations of quantum delayed-choice experiments [15-19], where the wave-particle response of the quantum system is determined by the state of a quantum ancilla, which is not itself a part of the interferometer but instead determines the action (or inaction) of one of the two beam splitters. In these experiments, the test system is entangled with the ancilla through the conditional action of the beam splitter, while the beam splitter remains a fully classical object. With our setup, we can also investigate the transition of the QBS to a classical beam splitter due to its intrinsic environmentally induced decoherence. As far as we know, this is the first interference experiment in which one beam splitter in an interferometer is in a coherent superposition of its active and inactive states. We note that our setup, using a temporally based Ramsey interferometer, does not permit the spacelike separation required for Wheeler's original gedanken experiment.

The rotation $R_{1}$ of the qubit is produced by the microwave field stored in the resonator, which is resonantly coupled to the qubit transition $|g\rangle \leftrightarrow|e\rangle$ with coupling strength $\Omega$. If the resonator is in the state $|\alpha\rangle$, a coherent microwave photon state with amplitude $\alpha$ and mean photon number $\langle n\rangle=|\alpha|^{2}$, the qubit exchanges energy with the resonator and Rabi oscillates between $|g\rangle$ and $|e\rangle$. For simplicity, we assume $\alpha$ is real and positive. When $\alpha \gg 1$, after an interaction time $t_{\alpha}=\pi /(4 \alpha \Omega)$, the qubit state is approximately $\left|\psi_{\alpha}\right\rangle=(|g\rangle-i|e\rangle) / \sqrt{2}$, with the resonator field left close to its original state $|\alpha\rangle$. Numerical simulation shows that the approximation is good even for moderate values of $\alpha$. This result has a simple qualitative explanation: When the resonator's coherent field is not too weak, its Poissonian photon-number distribution - and hence its state-is insensitive to a one-photon change. If the resonator is instead in its ground state $|0\rangle$, the rotation $R_{1}$ does not occur and the qubit remains in $|g\rangle$. The second pulse, $R_{2}(\theta)$, generated by a classical microwave pulse, performs the transformations $|g\rangle \rightarrow\left(|g\rangle-i e^{-i \theta}|e\rangle\right) / \sqrt{2}$ and $|e\rangle \rightarrow$ $\left(|e\rangle-i e^{i \theta}|g\rangle\right) / \sqrt{2}$. When acting on the state $\left|\psi_{\alpha}\right\rangle$, this results in the state

$$
\left|\psi_{w}\right\rangle=-i\left[\sin (\theta / 2) e^{i \theta / 2}|g\rangle+\cos (\theta / 2) e^{-i \theta / 2}|e\rangle\right] .
$$

The probability of measuring the qubit in $|e\rangle$ is then $\cos ^{2}(\theta / 2)$, showing the $\theta$-dependent interference associated with wavelike behavior. If $R_{1}$ did not occur, due to the microwave resonator being in its ground state $|0\rangle$, the final state after the second rotation, $R_{2}$, is

$$
\left|\psi_{p}\right\rangle=\left(|g\rangle-i e^{-i \theta}|e\rangle\right) / \sqrt{2} .
$$

The probability to be in $|g\rangle$ or $|e\rangle$ is $1 / 2$, representing particlelike behavior without the $\theta$-dependent interference effects.
Now we suppose that the resonator is instead initially prepared in the cat superposition state

$$
\left|\psi_{b, i}\right\rangle=\mathcal{N}(\cos \varphi|\alpha\rangle-\sin \varphi|0\rangle),
$$

where $\mathcal{N}=\left[1-e^{-|\alpha|^{2} / 2} \sin (2 \varphi)\right]^{-1 / 2}$. After the two rotations $R_{1}$ and $R_{2}(\theta)$, the qubit-QBS system will then approximately be in the entangled state

$$
\left|\psi_{q+b, f}\right\rangle \simeq \mathcal{N}_{t}\left(\cos \varphi\left|\psi_{w}\right\rangle|\alpha\rangle-\sin \varphi\left|\psi_{p}\right\rangle|0\rangle\right),
$$

where $\mathcal{N}_{t}=\left[1-e^{-|\alpha|^{2} / 2} \sin (2 \varphi) / \sqrt{2}\right]^{-1 / 2}$. The probability $P_{e}$ for finding the qubit in the state $|e\rangle$ is then close to

$$
P_{e} \simeq \mathcal{N}_{t}^{2}\left[\frac{1}{2} \sin ^{2} \varphi+\cos ^{2} \varphi \cos ^{2} \frac{\theta}{2}\right]
$$

The Ramsey interference pattern thus simultaneously exhibits the wave and particle behaviors, through the presence and absence, respectively, of $\theta$ dependence in the two terms that make up $P_{e}$. The quantum coherence between the two state components $|\alpha\rangle$ and $|0\rangle$ precludes the possibility of the qubit knowing in advance what type of experiment will be performed. We note that for $\varphi=0$ this interferometer is equivalent to the cavity QED analogue described in Ref. [5] except there the qubit is initially in $|e\rangle$.

The detailed implementation of this quantum delayedchoice experiment involves a superconducting resonator and two tunable superconducting phase qubits, one qubit serving as the test qubit and the second control qubit used to program the state of the superconducting resonator, as well as to read out the resonator at the end of each experiment. The two qubits are coupled to the resonator with on-resonance coupling strengths $\Omega$ and $\Omega^{\prime}$, respectively, and the effective coupling of each qubit to the resonator can be switched on or off by tuning the qubit on or off resonance with the resonator. The experimental apparatus is identical to that described in Ref. [24]. Using this apparatus, we can arrive at the state $\left|\psi_{b, i}\right\rangle$ of Eq. (3) with $\alpha=2$, and arbitrary values of $\varphi$ (Ref. [21]; see the Supplemental Material [25]). For $\varphi=\pi / 4$, the fidelity $F=\left\langle\psi_{b, i}\left|\rho_{b, i}\right| \psi_{b, i}\right\rangle=0.726 \pm 0.028\left(\rho_{b, i}\right.$ is the density operator corresponding to the output state). It would be preferable to obtain states with larger amplitude $\alpha$, but we are limited by the small nonlinearity of our phase qubits to $\alpha \lesssim 2$. Fortunately, numerical simulations show that the overlap between the final qubit-QBS state and $\left|\psi_{q+b, f}\right\rangle$ of Eq. (4) is higher than 0.98 even for this value of $\alpha$ (ignoring experimental imperfections). After generating $\left|\psi_{b, i}\right\rangle$, we resonantly couple the test qubit to the resonator for a time $t_{\alpha}$, and then apply a carefully tuned, on-resonance microwave $\pi / 2$ pulse to the qubit, thus implementing the rotation $R_{2}(\theta)$. Figure 2(a) displays the measured probability $P_{e}$ as a function of $\theta$ and $\varphi$, which clearly demonstrates the morphing between particle and wave behavior. To more precisely understand this behavior, we 
need to examine the state of the QBS. This is achieved using the control qubit, initially in the state $\left|g^{\prime}\right\rangle$, and tuning it into resonance with the resonator for a time $\pi /\left(2 \alpha \Omega^{\prime}\right)$. If the resonator contains a coherent field $|\alpha\rangle$, the control qubit will absorb a photon and undergo the transition $\left|g^{\prime}\right\rangle \rightarrow\left|e^{\prime}\right\rangle$, while if the resonator is in $|0\rangle$, the qubit remains in $\left|g^{\prime}\right\rangle$. The test qubit behavior is postselected by correlating its measurements with the outcomes of the control qubit measurements. We note that $|\alpha\rangle$ is not strictly orthogonal to $|0\rangle$, with the overlap $|\langle\alpha \mid 0\rangle|^{2} \approx 10^{-2}$ for $\alpha=2$, so that these two components cannot be unambiguously discriminated; there is a small probability that the detection of $|0\rangle$ actually comes from $|\alpha\rangle$. With $\alpha$ somewhat larger, this overlap will become negligible: For $\alpha=3$, the overlap is only about $10^{-4}$.

Figure 2(b) shows the measured probabilities $P_{e ; e^{\prime}}$ and $P_{e ; g^{\prime}}$ for detecting the test qubit in the state $|e\rangle$ conditioned upon the detection of the control qubit in $\left|e^{\prime}\right\rangle$ and $\left|g^{\prime}\right\rangle$, respectively; these are measured as a function of $\theta$. Here the parameter $\varphi$ is $\pi / 4$, corresponding to the QBS being in an equal superposition of its active and inactive states. As expected, $P_{e ; e^{\prime}}$ exhibits Ramsey interference fringes, with the contrast reaching 0.839 , while $P_{e ; g^{\prime}}$ remains almost constant. The slight oscillations in $P_{e ; g^{\prime}}$ are mainly due to the fact that the amplitude of the coherent state component $|\alpha\rangle$ is somewhat small, so that the control qubit has a small probability of remaining in the ground state after the interaction even when the resonator is in $|\alpha\rangle$. On one hand, the coherent state contains a vacuum state component, which is decoupled from the qubit state $\left|g^{\prime}\right\rangle$. On the other hand, not all the other superposed Fock states can make the control qubit flip to the excited state $\left|e^{\prime}\right\rangle$ with a unity probability
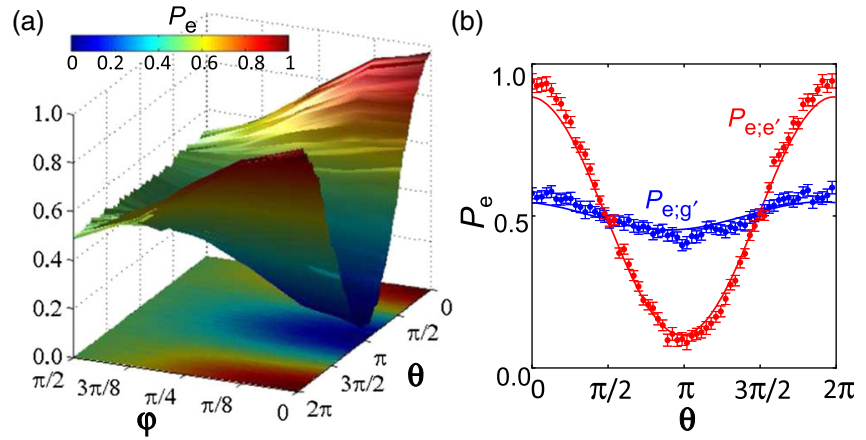

FIG. 2 (color online). Measured Ramsey interference signal. (a) Probability $P_{e}$, defined in Eq. (5), vs $\theta$ and $\varphi$. The amplitude of the coherent state component of the cat state $\left|\psi_{b, i}\right\rangle$ is $\alpha=2$. The transition between the wave and particle behaviors is clearly apparent. (b) Probabilities $P_{e ; e^{\prime}}$ and $P_{e ; g^{\prime}}$, vs $\theta$, for detecting the test qubit in $|e\rangle$ given that the control qubit is detected in $\left|e^{\prime}\right\rangle$ and $\left|g^{\prime}\right\rangle$, respectively. The control qubit's state reflects the QBS state, as this qubit is measured after it has interacted with the post- $R_{2}$ resonator state for a time $\pi /\left(2 \alpha \Omega^{\prime}\right)$. Data are for $\varphi=\pi / 4$. Error bars indicate the statistical variance. Lines are simulations taking into account imperfections in preparing $\left|\psi_{b, i}\right\rangle$. after the interaction, owing to the photon-number dependence of the Rabi frequency. These two reasons account for the $\theta$-dependent interference effect in $P_{e ; q^{\prime}}$, with a fringe contrast measured to be 0.19 . It is noted that taking into account the imperfect initial state as we prepared experimentally, we could verify that the measured fringe data (dots with error bars) are in good agreement with the numerical simulation (lines) in Fig. 2(b). With an increase in the coherent state amplitude $\alpha$, the state $|\alpha\rangle$ is more clearly distinguished from $|0\rangle$, and as a result the unwanted oscillations decay dramatically: For $\alpha=3$, the calculated fringe contrast is reduced to 0.048 .

It should be noted that the same statistical data can be produced if the resonator field is in the classical mixture $\cos ^{2} \varphi|\alpha\rangle\left\langle\alpha\left|+\sin ^{2} \varphi\right| 0\right\rangle\langle 0|$. To exclude the classical interpretation, the existence of quantum coherence between $|\alpha\rangle$ and $|0\rangle$ should be verified. The quantum state of the resonator field can be characterized by measuring its Wigner function (WF) $W(\chi)$, which describes the quasiprobability distribution of the microwave field in resonator phase space [23]. The WF associated with the density operator $\rho_{b}$ is defined as

$$
W(\chi)=\frac{2}{\pi} \operatorname{Tr}\left[e^{i \pi a^{\dagger} a} D(-\chi) \rho_{b} D(\chi)\right],
$$

where $\chi$ is the (complex) coordinate in phase space and $D$ is the displacement operator. This quantity is always nonnegative for the QBS in a classical mixture; the observation of negative values in regions of phase space is a signature of quantum interference. The Wigner function of the QBS was measured using the control qubit, following the procedure developed in Ref. [21]. In Fig. 3(a), we display the WF after performing the second rotation $R_{2}(\theta)$, but without reading out the state of the test qubit, while in Figs. 3(b) and 3(c) we show the WFs measured with the test qubit having been measured in $|g\rangle$ and $|e\rangle$, respectively, all for $\theta=\pi / 2$ and $\varphi=\pi / 4$. Simulated and measured WFs are shown in the upper and lower panels, respectively. Experimental imperfections are not included in the numerical simulations. Since the qubit wave state $\left|\psi_{w}\right\rangle$ is not orthogonal to the particle state $\left|\psi_{p}\right\rangle$, there is some quantum coherence between $|\alpha\rangle$ and $|0\rangle$, even when the test qubit is traced out. As a result, the WF exhibits a strongly nonclassical feature around $\chi=1$, as shown in Fig. 3(a). The measured $\mathrm{WF}$ has a minimum value of $-0.258 \pm 0.030$ at $\chi=0.84-0.03 i$. In Fig. 3(a), the shapes of the calculated and measured WFs agree well, demonstrating that the measured negative quasiprobabilities are due to quantum interference between $|\alpha\rangle$ and $|0\rangle$. The existence of quantum coherence between these two state components implies that their correlation with the behavior of the test qubit shown in Fig. 2(b) is nonclassical. Without reading out the test qubit's state, the WF is independent of the value of $\theta$ since any local unitary operation on the test qubit does not affect the QBS state after their interaction. When the test qubit's state is measured, the minimum value of the WF becomes 

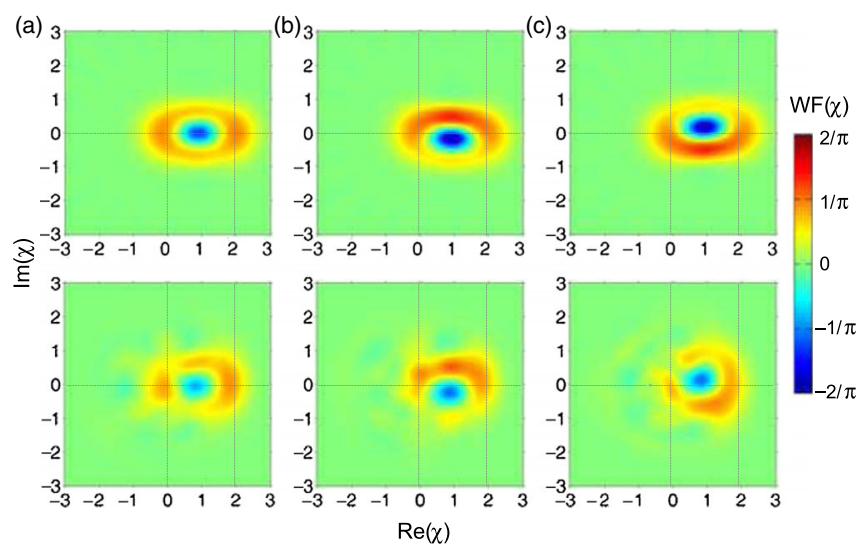

FIG. 3 (color online). Wigner tomography of the quantum beam splitter. The parameters are $\alpha=2, \theta=\pi / 2$, and $\varphi=\pi / 4$. The quantum beam splitter Wigner functions (WFs) are displayed for three cases: (a) the test qubit state is not read out; (b) the test qubit is measured in $|g\rangle$; (c) the test qubit is measured in $|e\rangle$. The simulated and measured WFs are shown in the upper and lower rows, respectively, as a function of the coordinate $\chi$ in resonator phase space. Experimental imperfections are not included in the numerical simulations. The minimum values of the three measured WFs are $-0.258 \pm 0.030,-0.342 \pm 0.027$, and $-0.336 \pm 0.028$, respectively.

more negative, implying the enhancement of quantum interference between the active and inactive states of the QBS. We have also measured the corresponding WFs for $\varphi=\pi / 8$ and $3 \pi / 8$ (see Fig. S.2 in the Supplemental Material [25] for details). As expected, for both cases there exists a region where the WF has negative values, and the quantum interference is enhanced when the test qubit's state is measured. These results further show that there exists entanglement between the quantum beam splitter and the test qubit. We note the final QBS-qubit state was realized deterministically and the qubit measurement was done in a single-shot manner. Another benefit of this experimental implementation is that it allows the observation of the transition from a quantum to a classical beam splitter, as shown in the Supplemental Material [25].

We have proposed and carried out a quantum delayedchoice experiment for a qubit interacting with a Ramsey interferometer, achieved by preparing one of the two Ramsey beam splitters in a superposition of its active and inactive states. Unlike previous experiments, the beam splitter here is really a quantum object, and the qubit behavior is clearly correlated with the quantum state of this beam splitter, significantly different from situations where an ancilla controls the transformation produced by a classical beam splitter. The quantum nature of the QBS is unambiguously verified by the negative values of its Wigner function. We have also observed variations in the Ramsey fringe contrast and the corresponding negativity of the Wigner function as a function of delay, illustrating the quick damping of the quantum coherence of the QBS. Using qubits with stronger nonlinearity and longer coherence times, we plan to increase the size and fidelity of the cat state and to explore the gradual transition from a quantum to a classical measuring device. This experiment was realized using a circuit QED system; however, similar experiments could be performed using microwave cavity QED [31,32] or ion-trap setups [33,34]. We further note that the idea of producing a conditional rotation on a qubit with an oscillator in a superposition state may be useful for the generation of important entangled states. These conditional dynamics, together with the qubitoscillator quantum state transfer [35], could be used to produce entanglement between two oscillators. Another example is the generation of entanglement between multiple qubits and an oscillator by performing rotations on these qubits conditional on the oscillator's state.

We thank D. J. Wineland for the fruitful discussions. This work was supported by the Major State Basic Research Development Program of China under Grants No. 2012CB921601, No. 2014CB921200, and No. 2012CB927404, and the National Natural Science Foundations of China under Grants No. 11374054, No. 11222437, and No. 11374083. The experiment was performed at Zhejiang University.

*t96034@fzu.edu.cn

†martinis@physics.ucsb.edu

*anc@uchicago.edu

[1] N. Bohr, in Quantum Theory and Measurement, edited by J. A. Wheeler and W. H. Zurek (Princeton University Press, Princeton, NJ, 1984), p. 9.

[2] M. O. Scully, B. G. Englert, and A. Walther, Nature (London) 351, 111 (1991).

[3] B. G. Englert, Phys. Rev. Lett. 77, 2154 (1996)

[4] S. B. Zheng, Opt. Commun. 173, 265 (2000).

[5] P. Bertet, S. Osnaghi, A. Rauschenbeutel, G. Nogues, A. Auffeves, M. Brune, J. M. Raimond, and S. Haroche, Nature (London) 411, 166 (2001).

[6] J. A. Wheeler, in Mathematical Foundations of Quantum Mechanics, edited by A. R. Marlow (Academic, New York, 1978), p. 9.

[7] J. A. Wheeler, in Quantum Theory and Measurement, edited by J. A. Wheeler and W. H. Zurek (Princeton University Press, Princeton, NJ, 1984), p. 182.

[8] A. J. Leggett, in Compendium of Quantum Physics, edited by D. Greenberger, K. Hentschel, and F. Weinert (Springer, Berlin, 2009), p. 161.

[9] T. Hellmuth, H. Walther, A. Zajonc, and W. Schleich, Phys. Rev. A 35, 2532 (1987).

[10] B. J. Lawson-Daku, R. Asimov, O. Gorceix, Ch. Miniatura, J. Robert, and J. Baudon, Phys. Rev. A 54, 5042 (1996).

[11] Y. H. Kim, R. Yu, S. P. Kulik, Y. Shih, and M. O. Scully, Phys. Rev. Lett. 84, 1 (2000).

[12] V. Jacques, E. Wu, F. Grosshans, F. Treussart, P. Grangier, A. Aspect, and J.-F. Roch, Science 315, 966 (2007).

[13] X. S. Ma, S. Zotter, J. Kofler, R. Ursin, T. Jennewein, C. Brukner, and A. Zeilinger, Nat. Phys. 8, 479 (2012). 
[14] R. Ionicioiu and D. R. Terno, Phys. Rev. Lett. 107, 230406 (2011).

[15] S. S. Roy, A. Shukla, and T. S. Mahesh, Phys. Rev. A 85, 022109 (2012).

[16] R. Auccaise, R. M. Serra, J. G. Filgueiras, R. S. Sarthour, I. S. Oliveira, and L. C. Celeri, Phys. Rev. A 85, 032121 (2012).

[17] J. S. Tang, Y. L. Li, C. F. Li, and G. C. Guo, Nat. Photonics 6, 600 (2012).

[18] F. Kaiser, T. Coudreau, P. Milman, D. B. Ostrowsky, and S. Tanzilli, Science 338, 637 (2012).

[19] A. Peruzzo, P. Shadbolt, N. Brunner, S. Popescu, and J. L. O'Brien, Science 338, 634 (2012).

[20] H. Lee, P. Kok, and J. P. Dowling, J. Mod. Opt. 49, 2325 (2002).

[21] M. Hofheinz, H. Wang, M. Ansmann, R. C. Bialczak, E. Lucero, M. Neeley, A. D. O'Connell, D. Sank, J. Wenner, J. M. Martinis, and A. N. Cleland, Nature (London) 459, 546 (2009).

[22] V. Buzek and P. L. Knight, in Progress in Optics Volume XXXIV, edited by E. Wolf (Elsevier, Amsterdam, 1995).

[23] W. P. Schleich, Quantum Optics in Phase Space (Wiley, Berlin, 2001).

[24] Y. P. Zhong, Z. L. Wang, J. M. Martinis, A. N. Cleland, A. N. Korotkov, and H. Wang, Nat. Commun. 5, 3135 (2014).

[25] See Supplemental Material at http://link.aps.org/ supplemental/10.1103/PhysRevLett.115.260403, which includes Refs. [26-30], for a discussion of the quantum circuit, the qubit-resonator interaction control, and details on implementation of $R_{1}$ and other experimental sequences, as well as a discussion of the observation of the transition from a quantum to a classical beam splitter.

[26] C. K. Law and J. H. Eberly, Phys. Rev. Lett. 76, 1055 (1996).

[27] H. Wang, M. Hofheinz, M. Ansmann, R. C. Bialczak, E. Lucero, M. Neeley, A. D. OConnell, D. Sank, M. Weides, J. Wenner, A. N. Cleland, and J. M. Martinis, Phys. Rev. Lett. 103, 200404 (2009).

[28] X. Y. LinPeng, H. Z. Zhang, K. Xu, C. Y. Li, Y. P. Zhong, Z. L. Wang, H. Wang, and Q. W. Xie, New J. Phys. 15, 125027 (2013).

[29] S. J. D. Phoenix, Phys. Rev. A 41, 5132 (1990).

[30] G. Kirchmair, B. Vlastakis, Z. Leghtas, S. E. Nigg, H. Paik, E. Ginossar, M. Mirrahimi, L. Frunzio, S. M. Girvin, and R. J. Schoelkopf, Nature (London) 495, 205 (2013).

[31] M. Brune, E. Hagley, J. Dreyer, X. Maitre, A. Maali, C. Wunderlich, J. M. Raimond, and S. Haroche, Phys. Rev. Lett. 77, 4887 (1996).

[32] S. Deleglise, I. Dotsenko, C. Sayrin, J. Bernu, M. Brune, J.-M. Raimond, and S. Haroche, Nature (London) 455, 510 (2008).

[33] C. Monroe, D. M. Meekhof, B. E. King, and D. J. Wineland, Science 272, 1131 (1996).

[34] C. Hempel, B. P. Lanyon, P. Jurcevic, R. Gerritsma, R. Blatt, and C. F. Roos, Nat. Photonics 7, 630 (2013).

[35] B. Vlastakis, G. Kirchmair, Z. Leghtas, S. E. Nigg, L. Frunzio, S. M. Girvin, M. Mirrahimi, M. H. Devoret, and R. J. Schoelkopf, Science 342, 607 (2013). 